\title{
EFEITOS DA TEMPERATURA SOBRE A ABSORÇÃO DE NUTRIENTES E CRESCIMENTO CELULAR DA MICROALGA THALASSIOSIRA WEISSFLOGII
}

LISANDRA I. MEINERZ ${ }^{1,2}$, EDUARDO L. C. BALLESTER ${ }^{1}$, LUCIANO JENSEN VAZ ${ }^{1}$ \& WILSON WASIELESKY JR. ${ }^{1}$

${ }^{1}$ Instituto de Oceanografia - Universidade Federal do Rio Grande - FURG. Cx. Postal 474, 96201-900 - Rio Grande - RS - Brasil. lisa_meinerz@yahoo.com.br

PALAVRAS CHAVE: Thalassiosira weissflogii, Densidade celular, Temperatura, Amônia total, Aquicultura.

As microalgas são amplamente utilizadas em atividades de cultivo, tanto para a alimentação direta de crustáceos, moluscos e peixes, como indiretamente, para a manutenção da qualidade da água (Amjad \& Jones 1994; Reitan et al. 1997). Elas desempenham papel fundamental no balanço do oxigênio, do dióxido de carbono, $\mathrm{pH}$ e na absorção das substâncias nitrogenadas (Gomes 1986; Hoff \& Snell 1987; Lavens \& Sorgeloos 1996). Possuem também função antibacteriana (Bruland 1983), através da produção de substâncias antibióticas (Reitan et al. 1997). Nas últimas décadas mais de cem espécies de microalgas foram testadas $e$ avaliadas para uso na aqüicultura, dentre estas, apenas aproximadamente vinte tiveram seu uso amplamente difundido (Brown et al. 1997; Olaizola 2003).

Durante a produção de microalgas em grande escala, deve haver um rígido controle do uso de fertilizantes ricos em produtos nitrogenados, necessários para o crescimento das células. Existem estudos que relatam o aumento nas concentrações dos nutrientes inorgânicos em tanques de larviculturas devido à introdução do uso de microalgas, o que poderia afetar o desempenho dos organismos cultivados (Tamaru et al. 1994; Jensen et al. 2006). Segundo estes autores, isto acontece devido à introdução de resíduos do meio de cultura das microalgas nos tanques de larvicultura, pois durante a sua produção são empregadas elevadas quantidades de fertilizantes ricos em fósforo e nitrogênio. Portanto, é necessário conhecer as condições em que o crescimento é potencializado, para que a produção atinja a densidade máxima no menor tempo possível, especialmente no que se refere aos principais fatores que controlam o crescimento das microalgas, principalmente luz, nutrientes, temperatura e pH (Tzovenis et al. 1997;
Zhu et al. 1997). Além disso, para que o problema dos altos teores de nitrogenados seja evitado, um controle efetivo do tempo de absorção destes nutrientes pelas microalgas deve ser realizado. Desta forma, o objetivo deste estudo foi avaliar a absorção de nutrientes (amônia total, nitrito e fosfato) durante o cultivo da diatomácea $T$. weissflogii em diferentes temperaturas.

O experimento foi realizado em uma estufa, onde as microalgas foram cultivadas durante 96 horas em duas diferentes temperaturas $\left(22^{\circ} \mathrm{C}\right.$ e $\left.27^{\circ} \mathrm{C}\right)$. Foram utilizados tanques retangulares de 1.000 litros, com três repetições para cada tratamento. $O$ volume inicial de cada tanque foi de 300 litros de água salgada filtrada (filtro de cartucho $\mathrm{Cuno}^{\circledR}, 5 \mu \mathrm{m}$ ). Para cada $100 \mathrm{~L}$ de água foram adicionados $15 \mathrm{~g}$ de sulfato de amônia $\left(\mathrm{NH}_{4}\right)_{2} \mathrm{SO}_{4} ; 0,75 \mathrm{~g}$ de uréia $\mathrm{CO}\left(\mathrm{NH}_{3}\right)_{2} ; 2,5 \mathrm{~g}$ de superfosfato triplo $3 \mathrm{Ca}$ $\left(\mathrm{H}_{2} \mathrm{PO}_{4}\right) \cdot 2 \mathrm{H}_{2} \mathrm{O}$ e $5,7 \mathrm{~g}$ de silicato de sódio neutro $\mathrm{Na}_{2} \mathrm{O} .3,3 \mathrm{SiO}_{2} \cdot \mathrm{H}_{2} \mathrm{O}$. O volume dos tanques foi elevado a cada 48 horas com a respectiva adição de nutrientes, até o volume final de 900 litros. A aeração foi mantida constante e moderada, o suficiente para manter o oxigênio dissolvido próximo a saturação e para evitar a sedimentação das células do cultivo. $O$ fotoperíodo foi natural, de aproximadamente $14 \mathrm{~h}$ claro/10 h escuro e a salinidade de 31. A densidade média inicial de $T$. weissflogii nos tratamentos 22 e 27 ${ }^{\circ} \mathrm{C}$ foi de $0,2 \times 10^{5} \mathrm{cel} / \mathrm{mL}$ e de $0,23 \times 10^{5} \mathrm{cel} / \mathrm{mL}$, respectivamente.

As respostas da microalga às condições testadas de temperatura foram avaliadas a partir da estimativa da densidade celular e da concentração de nutrientes no meio de cultivo, ao longo do tempo. Para o acompanhamento da absorção dos nutrientes, a cada 24 horas foram coletadas amostras de água do cultivo para a análise das concentrações de amônia total (UNESCO 1983), nitrito (Aminot \& 
Chaussepied 1983) e fosfato (Aminot 1977). Na mesma ocasião foram monitoradas a temperatura (termômetro de mercúrio, precisão de $0,5{ }^{\circ} \mathrm{C}$; a salinidade (refratômetro ótico ATAGO $^{\circledR}$ ); $0 \mathrm{pH}$ (pHmetro Handylab ${ }^{\circledR}$ 2BCN); e a luminosidade (luxímetro Chauvin Arnoux ${ }^{\circledR}$ CA 810). As estimativas de densidade celular $\left(\mathrm{n}^{\circ} \mathrm{0}\right.$ cel $\left.\times 10^{5} / \mathrm{mL}\right)$ foram determinadas também a cada 24 horas, a partir da contagem de células usando um hemocitômetro do tipo Neubauer Improved em um microscópio ótico de luz transmitida, binocular Nikon, com magnificação final de 100x. Para cada repetição de cultura, foram coletadas duas amostras para a estimativa da densidade celular, a partir das quais se calculou a média aritmética. A coleta de dados foi realizada sempre no mesmo horário (ao meio-dia) e, quando necessário, antes do aumento do volume dos cultivos (a cada 48h). A análise estatística dos resultados de crescimento celular e dos dados de qualidade de água colhidos durante $o$ experimento, foi realizada através de análise de variância unidirecional (ANOVA-ONEWAY) e quando determinadas diferenças significativas $(p<0,05)$, foi aplicado o teste 'a posteriori' de Tukey (Snedecor \& Cochran 1980).
As médias de temperatura nos tratamentos 22 ${ }^{\circ} \mathrm{C}$ e $27{ }^{\circ} \mathrm{C}$ foram $22,2 \pm 0,8$ e 27,4 $\pm 0,5^{\circ} \mathrm{C}$, respectivamente. A salinidade permaneceu constante (31) em todos os tanques e os valores médios de intensidade luminosa registrados na estufa foram $1.310 \pm 478$ lux. Ao final do experimento, a densidade celular média registrada nas temperaturas de $22{ }^{\circ} \mathrm{C}$ e $27 \stackrel{\circ}{\circ}$ foram respectivamente $1,22 \times 10^{5} \mathrm{cel} / \mathrm{ml}$ e de $3,2 \times 10^{5} \mathrm{cel} / \mathrm{ml}$ (Fig. 1) apresentando diferença significativa $(\mathrm{P}<0,05)$. Os valores médios de $\mathrm{pH}$ foram $8,52 \pm 0,06$ e $8,27 \pm 0,17$ para 22 e $27 \stackrel{\circ}{\circ}$, respectivamente, não havendo diferenças significativas entre os tratamentos $(P>0,05)$. $O$ mesmo ocorrendo para os valores das concentrações de nitrito $(0,016 \pm 0,005 \mathrm{mg} / \mathrm{L}$ e $0,02 \pm 0,01 \mathrm{mg} / \mathrm{L})$ e fosfato $(0,32 \pm 0,02 \mathrm{mg} / \mathrm{L}$ e $1,76 \pm 0,67 \mathrm{mg} / \mathrm{L})$ para as temperaturas 22 e $27 \stackrel{\circ}{\circ}$, respectivamente. Os resultados referentes à concentração final de amônia total (Fig. 2) demonstraram que no cultivo a $22{ }^{\circ} \mathrm{C}$ a absorção dos nutrientes foi menor, sendo a concentração de amônia encontrada $(24,86 \mathrm{mg} / \mathrm{L})$ significativamente maior $(P<0,05)$ que no cultivo em $27^{\circ} \mathrm{C}(9,15 \mathrm{mg} / \mathrm{L})$.

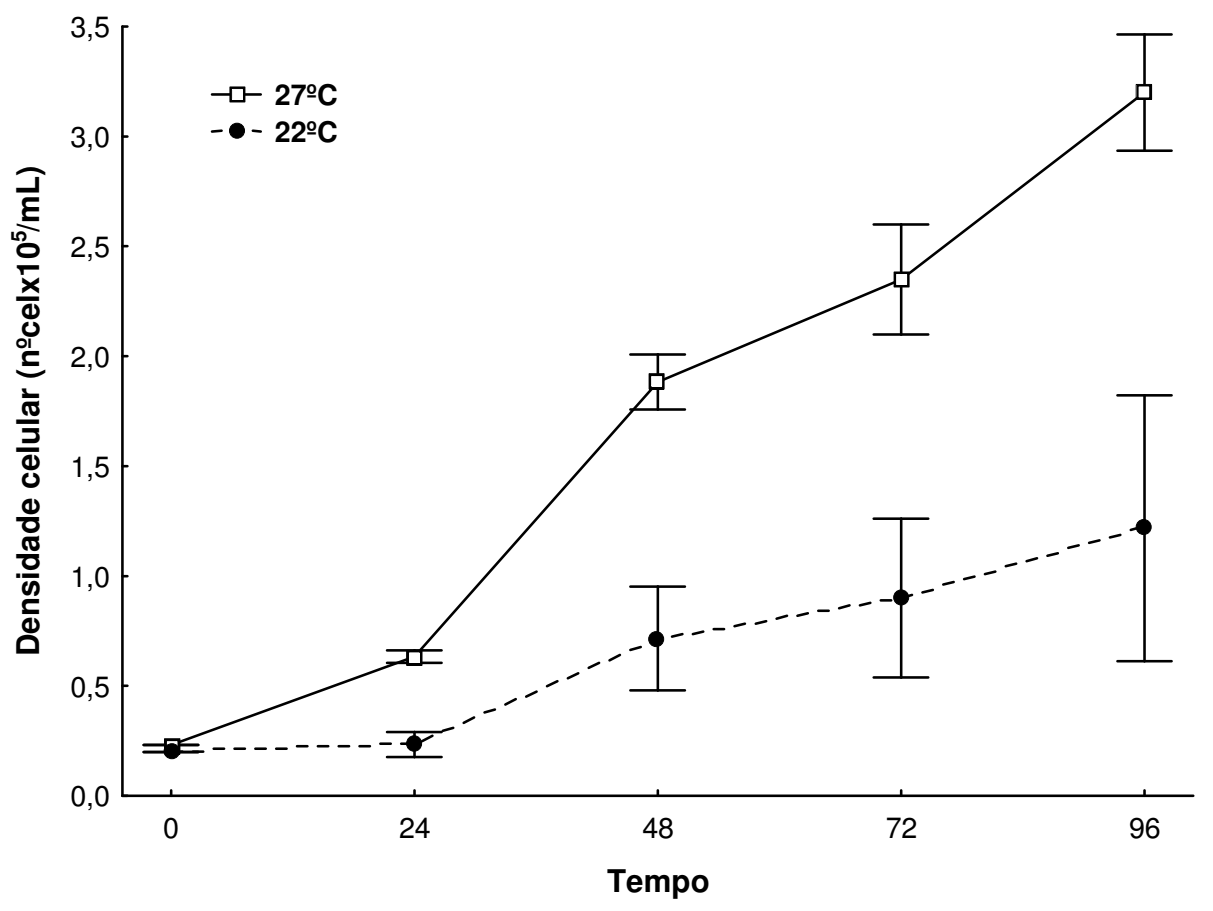

Figura 1: Médias $( \pm \mathrm{dp})$ da densidade celular $(\mathrm{cel} / \mathrm{mL})$ de Thalassiosira weissflogii nas temperaturas de $22^{\circ} \mathrm{C}$ e $27^{\circ} \mathrm{C}$ ao longo de 96 horas de experimento. $(\mathrm{N}=3)$. 


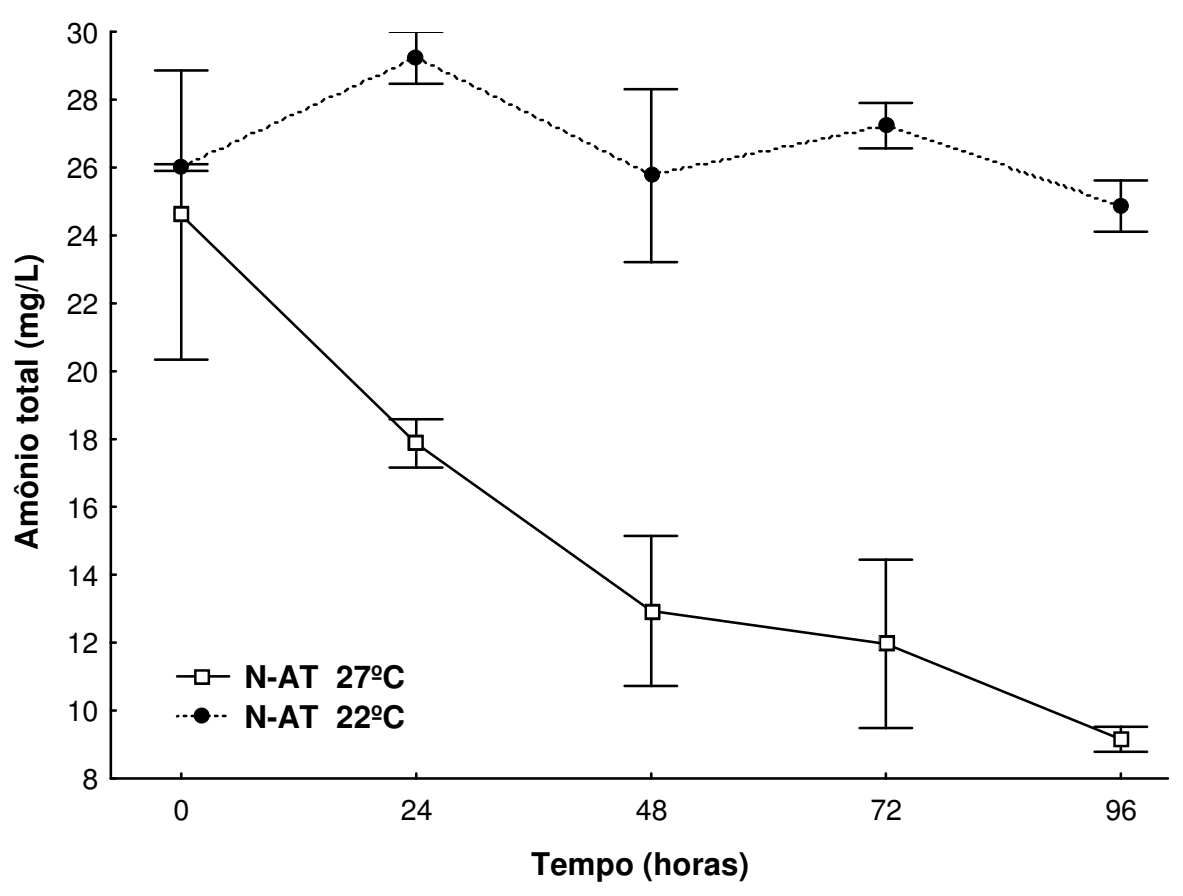

Figura 2: Médias ( $\pm \mathrm{dp}$ ) da concentração de amônia ( $\mathrm{mg} / \mathrm{L}$ ) total durante o cultivo de Thalassiosira weissflogii nas temperaturas de $22^{\circ} \mathrm{C}$ e $27^{\circ} \mathrm{C}$ ao longo de 96 horas de experimento. $(\mathrm{N}=3)$.

Os resultados do experimento demonstraram que a temperatura de cultivo teve influência direta na absorção de nutrientes pelas microalgas. A menor absorção dos nutrientes a $22 \stackrel{\circ}{\circ}$ ocorreu, provavelmente, devido à menor taxa de crescimento das microalgas cultivadas em temperatura reduzida. Entretanto, mesmo no cultivo a $27^{\circ} \mathrm{C}$, onde o crescimento foi considerado satisfatório, a concentração média final de amônia foi considerada elevada, visto que valores acima de $1 \mathrm{mg} / \mathrm{L} \mathrm{N}$-AT podem afetar o crescimento de larvas e pós-larvas de camarões peneídeos (Wasielesky et al. 1994; Van Wyk \& Scarpa 1999). Segundo Fogg \& Thake (1987) a maioria das microalgas cultivadas desenvolve-se entre 10 e $35 \stackrel{\circ}{\circ}$, com crescimento ótimo entre 20 e $25{ }^{\circ} \mathrm{C}$. Baixas temperaturas geralmente não matam as microalgas, mas acarretam redução das taxas de crescimento e divisão celular, fato que foi confirmado pelos resultados do presente experimento. Em temperaturas acima de $27{ }^{\circ} \mathrm{C}$, muitas microalgas apresentam queda na taxa de fotossíntese, perda no seu valor nutricional, podendo ocorrer elevada mortalidade devido ao comprometimento do metabolismo celular (Laing 1991). Estudos relatam que uma grande parte de espécies do gênero Thalassiosira cresce relativamente bem em baixas temperaturas e em baixas intensidades luminosas, como por exemplo, T. nordenskioldii (Popovich \& Gayoso 1999). Também foi demonstrado que a temperatura mínima que permite o crescimento de $T$. pseudonana é de $4{ }^{\circ} \mathrm{C}$ (Guillard \& Ryther 1962) e que $T$. rotula cresce bem a baixas intensidades luminosas e temperaturas moderadas (Krawiec 1982). Por outro lado, a faixa de temperatura na qual $T$. curviseriata cresce é de 5-20 ${ }^{\circ} \mathrm{C}$, no entanto o seu crescimento ótimo ocorre aos $20^{\circ} \mathrm{C}$ (Popovich \& Gayoso 1999), o que caracteriza esta espécie como euritérmica. Os resultados do presente experimento demonstraram que $T$. weissflogii cresceu bem em temperatura de 22 ${ }^{\circ} \mathrm{C}$, entretanto, com um aumento de $5^{\circ} \mathrm{C}$ na temperatura média houve uma aceleração significativa de seu crescimento, uma vez que a $27{ }^{\circ} \mathrm{C}$ a densidade celular alcançada foi três vezes maior que no tratamento a $22{ }^{\circ} \mathrm{C}$. Ou seja, T. weissflogii cresce em temperaturas mais elevadas que as reportadas para outras espécies do mesmo gênero, demonstrando possuir uma faixa de tolerância diferenciada. Além disso, ficou evidente que a absorção de nutrientes está diretamente relacionada com a taxa de crescimento e temperatura do cultivo para esta espécie de diatomácea.

A partir dos resultados obtidos no presente 
experimento é recomendado que o cultivo de $T$. weissflogii seja realizado na temperatura de $27^{\circ} \mathrm{C}$. Igualmente, é recomendado que seja avaliada a redução nas concentrações de nutrientes adicionados (principalmente a fonte de nitrogênio) e/ou o possível prolongamento do cultivo para que os níveis residuais de nutrientes sejam diminuídos e reduzam o risco de toxicidade para os organismos cultivados.

Também se sugere que estudos futuros sejam realizados, analisando o cultivo de $T$. weissflogii em temperaturas superiores as que foram avaliadas neste experimento.

\section{AGRADECIMENTOS}

Este estudo foi financiado pela FAPERGS, Pólo Pesqueiro e SCT-RS. W. Wasielesky Jr é bolsista de produtividade do CNPq.

\section{LITERATURA CITADA}

AMINOT, A. 1977. Manuel des méthodes de prélèvements et d'analyses. Caractéristiques physico-chimiques et hydrologiques. Réseau National d'Obsevation de la qualité du milieu marin, 1-142.

AMINOT, A \& M CHAUSSEPIED. 1983. Manuel des analyses chimiques en milieu marin. Brest: ANEXO. 395p.

AMJAD, S \& DA JONES. 1994. Role of microalgae in conditioning water in penaeid larval culture. Pakistan J. Mar. Sci., 3(1): 4151

BROWN, MR, SW JEFREY, JK VOLKMAN \& GA DUNSTAN. 1997. Nutricional properties of microalgae for mariculture. Aquaculture, 151: 315-331.

BRULAND, KW. 1983. Trace elements in sea-water. In: RILEY, JP \& R CHESTER (Eds) Chemical Oceanography. Academic Press, London, UK. Vol. 8: 157-220.

FOGG, GE \& B THAKE. 1987. Algal cultures and phytoplankton ecology. The University of Wisconsin Press, Madison, USA. $269 \mathrm{p}$.

GOMES, LAO. 1986. O cultivo de crustáceos e moluscos. Ed. Nobel. São Paulo, BR. 226p.

GUILLARD, RRL \& JH RYTHER. 1962. Studies of marine planktonic diatoms. L. Cyclotella nana Hustedt and Detonula confervacea (Cleve) Gran. Can. J. Microbiol., 8, 229-239.

HOFF, FH \& TW SNELL. 1987. Plankton Culture Manual. Florida Aqua Farms, Inc. Florida. $126 \mathrm{p}$.

JENSEN, LV, WJ WASIELESKY, ELC BALLESTER, RO CAVALLI \& MS SANTOS. 2006. Role of microalgae Thalassiosira fluviatilis in weight gain and survival of the shrimp Farfantepenaeus paulensis reared in indoor nursery tanks. Nauplius, 14 (1): 37-42.

KRAWIEC, RW. 1982. Autoecology and clonal variability of marine centric diatom Thalassiosira rotula (Bacillariophyceae) in response to light, temperature and salinity. Mar. Biol., 69: 7989.

LAING, I. 1991. Cultivation of marine unicellular algae. Ministry of Agriculture, Fisheries and Food, Directorate of fisheries research. Laboratory leaflet, $\mathrm{n}$ 667. Lowestoft, UK. 31p.
LAVENS, P \& P SORGELOOS. 1996. Manual on the production and use of live food for aquacultures. FAO Fisheries Technical Paper. № 361. Rome, FAO. 295p.

OLAIZOLA, M. 2003. Commercial development of microalgal biotechnology: from the test tube to the marketplace. Biomol. Engineering, 20: 459-466.

POPOVICH, CA \& M GAYOSO. 1999. Effect of irradiance and temperature on growth rate of Thalassiosira curviseriata Takano (Bacillariophyceae), a bloom diatom in Bahía Blanca estuary (Argentina). J. Plankton Res., 21(6): 1104-1110.

REITAN, KI, JRG RAINUZZO \& Y OLSEN. 1997. A review of the nutritional effects of algae in marine fish larvae. Aquaculture, 155: 207-221.

SNEDECOR, GW \& WG COCHRAN. 1980. Statistical Methods. Ames. lowa State. 507pp.

TAMARU, CS, R MURASHIGE \& CS LEE. 1994. The paradox of using background phytoplankton during the larval culture of striped mullet, Mugil cephalus L. Aquaculture, 119: 167-174.

TZOVENIS, L, N DE PAUW \& P SORGELOOS. 1997. Effect of different light regimes on the docosahexaenoic acid (DHA) content of Isochrysis aff. galbana (clone T-ISO). Aquacult. Int., 5: $489-507$.

UNESCO. 1983. Chemical methods for use in marine enviromental monitoring. Intergovernmental Oceanographic Commission. Manual and Guides 12. Paris. 53p.

VAN WYK, P \& J SCARPA. 1999. Water quality requirements and management. In: P VAN WYK et al. (editors). Farming Marine Shrimp in Recirculating Freshwater Systems. Tallahassee, Florida, USA. Florida Department of Agriculture and Consumer Services, pp. 128-138.

WASIELESKY, WJ, MA MARCHIORI \& MHS SANTOS. 1994. Efeito da amônia no crescimento de pós-larvas do camarão rosa, Penaeus paulensis, Pérez-Farfante, 1967 (Decapoda : Penaeidae). Nauplius, 2: 99-105.

ZHU, CJ, YK LEE \& TM CHAO. 1997. Effects of temperature and growth phase on lipid and biochemical composition of Isochrysis galbana TK1. J. Appl. Phycol., 9: 451-457. 